\section{Partner Companies and Subcontractors}

10.1. Due to the complex nature of the conflict/post-conflict environments, companies often employ the services of partner companies and subcontractors to fulfill the duties of their contract.

10.2. Signatories agree that they select partner companies and subcontractors with the utmost care and due diligence to ensure that they comply with all appropriate ethical standards, such as this Code of Conduct.

10.3. The future of the peace operations industry depends on both technical and ethical excellence. Not only is it important for IPOA member companies to adhere to the principles expressed in this Code, each member should encourage and support compliance and recognition of the Code across the industry.

\section{Application}

11.1. This Code of Conduct is the official code of IPOA and its member organizations. Signatories pledge to maintain the standards laid down in this Code.

11.2. Signatories who fail to uphold any provision contained in this Code may be subject to dismissal from IPOA at the discretion of the IPOA Board of Directors.

11.3. Member companies will endeavor to impart the basic principles of the IPOA Code of Conduct to their employees.

\section{Version: 11}

Adopted: December 1, 2006

Code of Conduct First Adopted: April 1, 2001

\title{
Neue Theorieansätze in der Friedens- und Konfliktfor- schung - Zweiter Workshop des AK Theorie der AFK
}

\section{Tagungsbericht}

\author{
Maurice Herchenbach ${ }^{1}$
}

Im Rahmen der Workshop-Reihe des Arbeitskreises Theorie der Arbeitsgemeinschaft für Friedens- und Konfliktforschung fand das zweite Arbeitstreffen vom 10. bis 12. April 2008 auf Schloss Rauischholzhausen bei Marburg statt. Wie zuvor im Juli 2007 fanden sich erneut zahlreiche Friedens- und KonfliktforscherInnen zusammen, die aus philosophischer, sprachwissenschaftlicher, soziologischer und politikwissenschaftlicher Perspektive neue Theorieansätze vorstellten und diskutierten (siehe die Homepage des Arbeitskreises unter http://www.uni-marburg.de/konfliktforschung/aktheorie).

Im Anschluss an die Eröffnung des Workshops skizzierte Christoph Weller einführend drei Dimensionen, entlang derer neue Entwicklungen erfasst werden können:

- Das frühere Ideal der friedenswissenschaftlichen Interdisziplinarität wird zunehmend von einer Multidisziplinarität abgelöst, die sich durch Eingebundenheit in eine „Hausdisziplin " und den intensiven Austausch zwischen den Disziplinen auszeichnet.

- Zahlreiche neue Gegenstände, die vor einigen Jahren noch wenig relevant erschienen, sind in den Forschungsfokus gerückt (z.B. Terrorismus, Friedenskonsolidierungsprozesse, fragile Staatlichkeit).

- Mit dem gestiegenen Einfluss der Friedens- und KonfliktforscherInnen auf die Politik, wie sich $u$. a. beim Zivilen Friedensdienst aber auch beim Demokratisierungsdiktum gezeigt hat, ist auch die Pflicht und Verantwortung zur eigenen Interventionsbeobachtung und -reflexion deutlich gestiegen.

1 Dipl.-Pol., Arbeitsbereich Internationale Friedens- und Sicherheitspolitik, Freie Universität Berlin.
Der Workshop begann mit einem Beitrag von Gertrud Brücher zu „Eskalation als Gewalt- und Friedensparadigma“. Darin unternimmt die Autorin den Versuch, Gewalt konzeptionell als Wechselwirkung zu erfassen, wobei sie unter Wechselwirkung Prozesse versteht, in denen sich Gewalt autopoietisch reproduziert. Wo Wechselwirkungen entstehen, verschwinden laut Brücher die Akteure der Gewalt. Gerade solche Gewalt aber sollte als zentrales Problem der Friedens- und Konfliktforschung erfasst werden, da für diese Gewaltprozesse bislang keine Deutungsmuster vorhanden sind. Eine herkömmliche, akteurszentrierte theoretische Perspektive, die weiterhin von der Unterscheidbarkeit von Tätern und Opfern ausgeht, ist Brücher zufolge nicht in der Lage zu erfassen, wo und wann vermeintlich nützliche Gewalt (aus der Perspektive der Akteure) eskaliert und nichtintendierte Effekte hervorbringt.

Zahlreiche Diskussionsbeiträge problematisierten die Definition von Konflikt als gewalthaltige Extremsituation, weil durch sie theoretische Erkenntnisse und praktische Nutzbarmachungen ausgeblendet würden, die auf einem Verständnis von Konflikt als Normalfall menschlicher Interaktion basierten. Vor diesem Hintergrund wurde die Frage gestellt, ob ein solcher Konfliktbegriff für die Friedenstheorie ausreiche. Darüber hinaus wurde festgestellt, dass ein systemtheoretischer Ansatz hinsichtlich des Verständnisses komplexer Konfliktsysteme zwar analytisches Potential besitze, gleichzeitig aber die Erkenntnisse der akteurszentrierten, sozialwissenschaftlichen Konfliktforschung nicht völlig vernachlässigt werden sollten.

Der Beitrag von Jörg Lehmann zu „Hate Speech in den Medien“ untersucht aus sprachtheoretischer Perspektive das Phänomen von Hassreden am Beispiel der Botschaften Osama bin Ladens. 
Das Erkenntnisinteresse richtet sich sowohl auf ein Verstehen des Gewaltvollzuges mittels Sprache als auch auf die Effekte, die Hassreden bei Zuhörern und Adressaten auslösen.

Im Fokus der Diskussion stand zunächst die Behauptung des Autors, dass Hassreden eine desorientierende Wirkung auf ihre Zuhörer hätten. Die Prämisse, dass Empfänger grundsätzlich andere Orientierungen als die Sprecher haben, wurde in ihrer Generalisierbarkeit angezweifelt. Auch seien die Massenmedien zu statisch erfasst und müssten hinsichtlich ihres eigenen Anteils bei der Wirkung von Hassreden analysiert und theoretisiert werden. Zudem wurden die von Lehmann verwendeten theoretischen Ansätze, etwa von John Austin und Judith Butler, als zu unidirektional für die Analyse eines genuin dialogischen Phänomens kritisiert. Die Auswahl von Reden Osama bin Ladens wurde als problematisch erachtet, da sie eher als Beispiele für Prophezeiungen als für Hassreden begriffen werden müssten.

Aus einer politisch-theoretischen Perspektive unternahm Alfred Hirsch in seinem Beitrag „Vorbemerkungen zu einigen Gewaltformen des Terrorismus“ den Versuch einer konzeptionellen Klärung des Phänomens des transnationalen Terrorismus. Terroristen werden von ihm im Sinne Carl Schmitts als postmoderne Partisanen charakterisiert, die sich durch ihre Irregularität, einen tellurischen Charakter (Erd- und Raumverbundenheit), eine gestiegene Mobilität sowie enormes politisches Engagement auszeichnen. Wie der Partisan so sei auch der Terrorist konstitutiv verknüpft mit der Existenz des Staates. Hieraus folgt, dass die Ur-Ursache des Terrors im Staat selbst liegt, der durch sein Gewaltmonopol terroristisches Handeln erst ermöglicht und bedingt.

Die Diskussionsbeiträge konzentrierten sich auf die Frage, inwieweit durch eine Beschreibung transnationaler Terroristen als Partisanen ein analytischer Mehrwert erzielt werden kann. Dabei wurde besondere die Brauchbarkeit des tellurischen Charakters in Frage gestellt. Zu bedenken wurde gegeben, dass diese gedankliche Figur von Carl Schmitt nicht übernommen werden könne, ohne auch dessen Blut-und-Boden-Romantik mitzunehmen. Aus historischer Perspektive sei der tellurische Charakter des Terroristen/Partisanen ohnehin kein überzeugendes Abgrenzungskriterium, da zahlreiche Rebellionen und soziale Bewegungen seit dem Zweiten Weltkrieg bereits entterritorialisierte Elemente enthalten hätten. Aus epistemologischer Perspektive wurde in Zweifel gezogen, ob die Erkenntnisse des okzidentalen politischen Theologen Schmitt überhaupt auf orientale theologische Phänomene übertragbar sind.

Matenia Sirseloudi versuchte in ihrem Papier „Lokaler Konflikt im globalen Jihad. Kontextualisierung innerstaatlicher und internationaler Konflikte in die Ideologie des transnationalen Jihad" eine Erklärung zu geben, warum Menschen zu terroristischer Gewalt greifen. Im Fokus des Papiers steht die Strukturanalyse einer zeitgenössischen jihadistischen Ideologie, deren Anknüpfungsfähigkeit an unterschiedliche lokale, nationale und regionale Identitäts- und Konfliktlinien den global zu beobachtenden Zuspruch zu dieser Ideologie erklärt. Für den gegenwärtigen Jihad wird konstatiert, dass der religiöse nicht nur dem politischen Frame vorgelagert sei, sondern auch die entscheidende Gemeinsamkeit aller im globalen Jihad vereinigten Gruppen darstelle.
Unter methodologischen Gesichtspunkten stellte sich für das Plenum grundlegend die Frage, ob Ideologie tatsächlich als Erklärungsvariable behandelt werden kann oder eher als allgemeine Hintergrundbedingung zu fassen sei. Zur Erklärung der Varianz bei der Unterstützung terroristischer Gruppen würden über diese hinaus weitere Bedingungen und Mechanismen benötigt. Sehr kritisch wurde beurteilt, dass die Autorin ideologische und religiöse Texte interpretiert, ohne selbst des Arabischen mächtig zu sein.

Weiter vertieft wurde die Debatte über den gegenwärtigen Terrorismus durch einen Beitrag von Michael Hörter zu „Terrorismusbekämpfung als friedensethische Herausforderung“. Darin geht der Autor der Frage nach, warum die (christliche) Friedensethik sich bislang noch nicht dem Thema Terrorismusbekämpfung gewidmet hat. Er erklärt dies damit, dass ein undifferenzierter Gewaltbegriff eine derartige Beschäftigung mit dem Thema bislang verhindert habe, was anhand zentraler Dokumente der beiden christlichen Kirchen Deutschlands aufgezeigt wird. Die dort verwendeten Gewaltbegriffe werden von Hörter als zu undifferenziert bezeichnet, weil sie nicht zwischen legitimen und illegitimen Gewaltformen unterscheiden. Er plädiert für einen Gewaltbegriff, der es ermöglicht, legitime Formen von Gewalt in den Friedensbegriff zu integrieren.

In der Diskussion wurde dem Autor vorgehalten, er bleibe mit der Forderung nach einer friedensethischen Grenzziehung zwischen legitimer und illegitimer Gewalt um Jahre hinter der friedenstheoretischen Debatte zurück. In dieser sei die Möglichkeit einer solchen Unterscheidung nicht nur für obsolet erklärt, sondern auch die Rechtfertigung staatlicher bzw. revolutionärer Gewalt als Gefahr für den Friedensbegriff analysiert worden. Durch den Versuch der Legitimierung von Gewalt stelle sich in besonderer Weise die Frage, was Friedensethik von „normaler" Ethik unterscheide.

Martin Quacks Papier zu „Wirkungsanalysen in der Friedensförderung: Theoretische Bezüge" analysiert die Notwendigkeit von kausalen und normativen Theorien zur Bewertung der Wirkungen von Interventionen externer Akteure, speziell solcher im Rahmen der zivilen Konfliktbearbeitung. Das Papier greift ein Modell aus der Entwicklungszusammenarbeit auf, bei dem die Analyse über die Wirkungskette von Input über Output und Outcome hin zum Impact angeleitet wird. In diesem Modell sind auch die unterschiedlichen Ebenen und Wechselwirkungen von Interventionsakteuren und lokalen Partnern enthalten.

In der Diskussion wurde besonders hervorgehoben, dass der Beitrag dem bisherigen Mangel an theoretischen Konzepten zur Analyse der Wirkung externer Friedensarbeit begegnet. Der gestiegenen Nachfrage nach Wirkungsanalysen, sowohl von Seiten der Wissenschaft als auch der Politik, sei bislang noch unzureichend begegnet worden. Es bleibe aber die Herausforderung, plausible und adäquate Indikatoren zu bilden. Vorgeschlagen wurde die Entwicklung von Prozessindikatoren, die, anders als statische Einzelindikatoren (bspw. zur Erfassung des sozioökonomischen Entwicklungsstandes), eine angemessene Grundlage für die Beobachtung von Wirkungen in gesellschaftlichen Prozessen zu bilden in der Lage sind. Als notwendige Grundlage zur Analyse von Wandel fehle dem Papier aber eine Theorie der Gewaltwirkungen, durch die die „Schädigungen“ 
in dem sozialen Gefüge zu erkennen wären, auf die die zivile Konfliktbearbeitung einwirke.

Zum Abschluss der Tagung stellte Ariel Macaspac Penetrante sein Papier „Guns, Goons, and Gold as Burdens of a Fragile State - Dynamics and Mechanisms of SALW and Internal Conflict in the Philippines"vor. Darin untersucht der Autor anhand einer Studie zu den Philippinen den Zusammenhang zwischen schwacher Staatlichkeit und der Proliferation von Kleinwaffen. Als entscheidende Faktoren identifiziert er die horizontale und vertikale Gewaltenteilung sowie eine dominante männliche Waffenkultur.

Kommentar und Diskussion konzentrierten sich zum einen auf begriffliche Unklarheiten, fehlende Definitionen und den Verzicht auf einen friedenstheoretischen Bezugsrahmen. Weiterhin wurde die Verwendung eines westlichen Staatsbegriffs bei der Analyse nicht-europäischer bzw. nicht-westlicher Regionen problematisiert. Aus normativer Perspektive wurde in diesem Zusammenhang auf die Gefahren hingewiesen, die daraus erwachsen, den Staat als Ordnungsform absolut zu setzen und sein Potential zur Friedensgefährdung nach innen zu vernachlässigen.

Trotz der Heterogenität der theoretischen Ansätze und empirischen Phänomene, mit denen sich die Beiträge des Workshops beschäftigen, gelang es ihm deutlich besser als dem vorhergehenden, die Diskussionen auf die übergeordneten
Theoriefragen der Friedens- und Konfliktforschung zu beziehen. Es kristallisierten sich neben der anhaltenden Debatte über die Vereinbarkeit systemtheoretischer und handlungstheoretischer Ansätze für die Friedenstheorie drei wichtige Theorieprobleme heraus:

- Erstens ist das Verhältnis von Staat und Gewalt nach wie vor umstritten. Es scheint die Gemeinschaft der Friedens- und KonfliktforscherInnen weiter bezüglich der Bedeutung des Missbrauchs- und Entgrenzungspotentials staatlicher Gewaltmonopole auf der einen und der Anerkennung der friedensschaffenden Wirkung staatlicher Herrschaft auf der anderen Seite zu teilen.

- Zweitens bleiben theoretische Defizite bei der Erfassung der Interventionsproblematik sowohl in normativer (Legitimität und Legalität militärischer, ziviler, entwicklungspolitischer Interventionsentscheidungen) als auch in empirischer Hinsicht (Analyse der komplexen Interaktion von Strukturen, Prozessen und Akteuren).

- Insbesondere die Analyse von Hassreden, Massenmedien und Legitimationsmustern am Beispiel des Terrorismus bzw. der Terrorismusbekämpfung verdeutlichte drittens die zentrale Rolle von Sprache und Kommunikation bei Eskalations- und Deeskalationsprozessen in Konflikten sowie die mangelhafte Integration entsprechender Ansätze in die Theoriebildung der Friedens- und Konfliktforschung.

\title{
Macht, Recht, Frieden ${ }^{1}$
}

\author{
Egon Bahr ${ }^{2}$
}

1984 konzentrierte sich das ISFH auf den Bericht der PalmeKommission, der unter dem Titel „Gemeinsame Sicherheit“ zu der damals noch ungewohnten und umstrittenen Erkenntnis gekommen war, dass im Zeitalter gegenseitig gesicherter Zweitschlagsfähigkeit durch strategische Atomwaffen Sicherheit nur mit dem potenziellen Gegner zu erreichen ist und nicht mehr gegen ihn. In der damals bipolaren Welt konnten sich nicht nur die Regierungen, sondern auch die Wissenschaftler ein Ende der Sowjetunion nicht vorstellen.

Die Deutsche Einheit, die Implosion der Sowjetunion, das Ende des Wahrschauer Paktes beendete die Blockkonfrontation. Das Risiko eines apokalyptischen Krieges sankt gegen Null, aber die Realität vieler kleiner Kriege rückte die Vision eines dauerhaft gesicherten Friedens in unbestimmte Ferne. Aus der Blockdisziplin entlassen, schienen begrenzte Gewaltanwendungen wieder kalkulierbar. Das ISFH stellte fest: „Vier Jahre seit der säkularen Zäsur von 1989 zählt Europa ein vielfaches an Kriegstoten als in den vier Jahrzehnten der Ost-West-Konfrontation.“ Das Ergebnis unserer Überlegungen - in Anknüpfung an das kollektive Grundkonzept der UN - veröffentlichten wir 1993, ein Modell für eine „Europäische Sicherheitsgemeinschaft als

1 Manuskript eines Vortrages an der Universität Hamburg am 8.7.2008 zum Gedenken an Dieter S. Lutz, ehemaliger Direktor des IFSH und Begründer dieser Zeitschrift.

2 Bundesminister a.D., ehemaliger Direktor des IFSH.
Garant für Sicherheit und Frieden“. Die Leitidee meinte die Ablösung des Rechts des Stärkeren durch die Stärke des Rechts. Diese Maxime ist seither international geläufig geworden. Das Urheberrecht hat meiner Erinnerung nach eine Mitarbeiterin des Instituts. Der Spannungsbogen von Macht über Recht zum Frieden ist wie man sieht, bis heute aktuell geblieben.

Die Welt des Jahre 2008 ist mit der vor 18 Jahren kaum mehr vergleichbar. Russland ist nach einer Periode chaotischer Schwäche zu nationalem Selbstbewusstsein zurückgekehrt, basierend auf seinem Energiereichtum bei wachsendem Weltmarktbedarf und steigenden Preisen. Es ist das einzige Land, das über die atomare Zweitschlagsfähigkeit verfügt, obwohl die Abschreckung theoretisch weiter gilt, die Washington und Moskau pflegen, ohne von ihr abhängig zu sein. China ist Weltmacht geworden mit einer Wirtschaftskraft, die in überschaubarer Zeit die der USA übertreffen kann. In der Entwicklung seiner modernen Technologie steht Indien kaum nach. BRIC ist eine geläufige Abkürzung für die Giganten Brasilien, Russland, Indien und China geworden, die ihre Interessen abzustimmen begonnen haben. Amerika, Europa und Japan gehören nicht dazu. Klimaerwärmung und Bewahrung der Umwelt haben sich zu dem großen übergeordneten Thema des neuen Jahrhunderts entwickelt, sie werden die Weltmächte mittelfristig zu einer Zusammenarbeit nötigen, von der wir einen Vorge- 